OPEN ACCESS

Edited by:

Rahul Shidhaye,

Pravara Institute of Medical

Sciences, India

Reviewed by:

Rüdiger Christoph Pryss,

Julius Maximilian University of

Würzburg, Germany

Simona Pajaujiene,

Lithuanian Sports University, Lithuania

*Correspondence:

Hongli Yu

hongli.yu@awf.gda.pl

Specialty section: This article was submitted to

Digital Public Health,

a section of the journal

Frontiers in Public Health

Received: 01 December 2021

Accepted: 24 January 2022

Published: 17 February 2022

Citation:

Yu $\mathrm{H}, \mathrm{He}$ J, Wang $X$, Yang W, Sun $B$ and Szumilewicz $A$ (2022) $A$

Comparison of Functional Features of Chinese and US Mobile Apps for Pregnancy and Postnatal Care: A

Systematic App Store Search and

Content Analysis.

Front. Public Health 10:826896. doi: 10.3389/fpubh.2022.826896

\section{A Comparison of Functional Features of Chinese and US Mobile Apps for Pregnancy and Postnatal Care: A Systematic App Store Search and Content Analysis}

\author{
Hongli $\mathrm{Yu}^{1,2 *}$, Juan $\mathrm{He}^{1}$, Xinghao Wang ${ }^{1}$, Weilin Yang ${ }^{1}$, Bo Sun ${ }^{1}$ and Anna Szumilewicz ${ }^{1}$ \\ ${ }^{1}$ Department of Sport, Gdańsk University of Physical Education and Sport, Gdańsk, Poland, ${ }^{2}$ Jiuling Primary School, \\ Mianyang, China
}

Background: Pregnancy to postpartum (PtP) applications (apps) are becoming more common tools to document everything from pregnancy and delivery to nutrient allocation, life taboos, and infant medical examinations. However, the dependability, quality, and efficacy of these apps remain unclear. This study examined the features and functions of mobile PtP care apps accessible in China and the United States and to identify the major gaps that need to be addressed.

Methods: Apps were selected by searching the Apple App Store and Android Markets (in the US and China) for the terms "pregnancy" and "postpartum" in Chinese and English. The apps' security, quality, and effectiveness were investigated, and chi-square tests and analysis of variance were performed to examine the differences in characteristics between apps available in the US and China.

Results: A total of 84 mobile PtP care apps (45 from the US and 39 from China) were included. A total of $89.7 \%$ (35/39) of Chinese mobile apps did not provide safety statements or supporting evidence. The objective app quality ratings for Chinese and US apps were $3.20 \pm 0.48$ (mean \pm standard deviation) and $3.56 \pm 0.45$, respectively $(p>0.05)$. A greater number of Chinese apps provided app-based monitoring functions, namely recording fetal size ( $n=18,46.2 \%$ in China vs. $n=3,6.7 \%$ in the US), contractions ( $n=11,28.2 \%$ in China vs. $n=0,0 \%$ in the US), pregnancy weight ( $n=11$, $28.2 \%$ in China vs. $0,0 \%$ in the US), and pregnancy check-up reminders ( $n=10,25.6 \%$ in China vs. $n=0,0 \%$ in the US). Meanwhile, a greater number of US apps provided exercise modules, namely pregnancy yoga ( $n=2,5.1 \%$ in China vs. $n=21,46.7 \%$ in the US), pregnancy workouts ( $n=2,5.1 \%$ in China vs. $n=13,28.9 \%$ in the US), and pregnancy meditation ( $n=0,0 \%$ in China vs. $10,22.2 \%$ in the US) $(p<0.01)$. A medium security risk was identified for $40 \%$ (18/45) of apps in the US and $82.1 \%(32 / 39)$ of apps in China $(p<0.01)$.

Conclusions: The functionality and characteristics of in-store mobile apps for PtP care varied between China and the US. Both countries' apps, particularly Chinese apps, encountered issues related to a lack of evidence-based information, acceptable 
content risk, and program evaluations. Both countries' apps lacked proper mental health care functions. The findings suggest that the design of app features should be enhanced in both countries, and increased interaction between app creators and users is recommended.

Keywords: pregnancy, postnatal care, mobile app, China, functionalities, United State of America

\section{INTRODUCTION}

The pregnancy to postpartum (PtP) stages are critical because, during this period, a woman's health is very vulnerable $(1,2)$. The 22nd Annual Meeting of the International Federation of Obstetrics and Gynecology discussed the present condition of PtP health care throughout the world. Experts noted that health care during the PtP period is imperative and encompasses a range of health care measures for the mother and fetus before, during, and after pregnancy, as well as during the puerperium (lactation) and neonatal phases (3).

During the PtP period, everything from pregnancy and delivery to nutrient allocation (4), life taboos (5), and newborn physical examinations (6) must be closely monitored. Pregnancy and postnatal education and support provided by a multidisciplinary team of specialists (e.g., doctors, midwives, trainers, and psychotherapists) may enhance the quality of care $(7,8)$. However, they may be too expensive or unavailable in impoverished countries $(9,10)$. Additionally, coronavirus disease 2019 (COVID-19) and its corresponding isolation periods may harm the health of pregnant and postpartum women (11). Thus, a lack of professional coaches, financial support, and infrastructure may hinder the provision of PtP care from a multidisciplinary team of specialists.

Technological advancements have facilitated the rapid rise of electronic health (eHealth) and mobile health (mHealth) in recent years $(12,13)$. According to a recent survey in Switzerland, PtP applications (apps) are becoming increasingly popular (14): $91 \%$ of parents use digital media to learn about their child's health and development (15). Evidence from randomized trials and comprehensive reviews indicates that mobile apps are typically beneficial for improving maternal physical health (e.g., weight management, mental health, and pregnancy awareness) (16-18), and affordability is a major advantage of such apps (19). However, the programs available in app stores are highly varied in terms of function, design, and overall quality, and they are not always subjected to rigorous evaluation through effective randomized controlled trials (RCTs) $(18,19)$. Meanwhile, systematic reviews of RCTs on maternal apps described the information for software and hardware, intervention content and delivery, and limitations. They still highlighted the risks of RTCs, such as unclear allocation concealment, no evaluation of objective quality in intervention apps, and no published protocols (20-22). Furthermore, studies reviewing apps that cover children's first 1,000 days of life (from conception to the age of 24 months) have exclusively focused on the prenatal or postnatal phases, ignoring the continuity between the two periods and their combined effect on the health of the mother and child $(23,24)$. For instance, taking notes of medical treatment received by both mother and infant, communicating with health professionals, as well as monitoring the mother's sleep and mental health are all uncommon features in apps (25). The dependability, quality, and efficacy of currently available PtP care apps are unclear, which may be a barrier to health promotion because pregnant and postpartum women are more susceptible to external influences (e.g., media coverage, apps information, social variables) (26). Incorrect information on health care and lifestyle may lead to unnecessary worry or stress during the perinatal period $(26,27)$.

Unsurprisingly, however, data supporting the effectiveness of mobile apps (26) are lacking because of the wide range of properties, responsibility for information correctness, degree of trustworthiness, and accessibility of content updates, as well as the absence of a certification mechanism or their categorization as a medical device (28). Furthermore, conventional app design and assessment procedures ignore the health literacy level of the target demographic and are disconnected from users' actual requirements $(29,30)$. Users' decisions may be influenced by variables such as an app's popularity, aesthetics, functionality, and user engagement $(29,30)$. However, one study revealed that many users did not critically evaluate the authenticity of the content offered by apps or consider problems related to their personal information and data (31).

Based on a review of recent studies of the impact of mHealth on the PtP period, two results emerged: (1) the importance of mothers receiving accurate health information throughout their children's first 1,000 days of life $(2,17)$ the major influence of mHealth on maternal well-being, lifestyle, and decision-making about pregnancy and infant health (32). Given these considerations, as well as the substantial gaps in the current research, several factors are unknown: (1) the authenticity, quality, and effectiveness of the most recent upgraded content provided by PtP care apps; (2) whether the apps consider privacy and security issues when collecting personal information and data. Therefore, a comprehensive study of current PtP care apps is timely and necessary during the COVID-19 pandemic.

Thus, the purposes of this study were to (1) describe and analyze the features and functions of mobile apps for PtP care available in China and the US, two of the largest app markets; (2) examine the apps' security, quality, and effectiveness; and (3) provide suggestions for future development and usage of mobile apps for PtP care of mothers and children throughout the first 1,000 days of life. On the basis of these goals, we anticipated that (1) the functions and features of PtP care apps in the US and China would be similar; (2) the latest upgraded content provided by apps would be high-quality and effective; and (3) all the apps would be concerned with the privacy and security of users' personal information and data. 


\section{MATERIALS AND METHODS}

\section{Data Source}

An electronic search of apps was performed from June 11 to August 5, 2021, using the keywords "pregnant woman, 9/9 months of pregnancy," "birth," "infant," "baby," "obstetrics," "pregnancy," "postpartum," "new baby," and "kid" in both English and Chinese languages. We selected English- or Chineselanguage apps from the Apple App store (in China and the US), Google Android Play (in the US), Huawei Android Market (Huawei Holdings Limited, in China), Baidu Android Market (Baidu, Inc., in China), and 360 Android Market (Qihoo 360 Technology Co., Ltd, in China).

\section{App Selection Criteria and Data Extraction}

The mobile apps for PtP care included in this study were defined as those that fulfilled any PtP health care needs. This study only included mobile apps with a minimum of 1 million downloads in the initial search list. Users are less likely to choose and download mobile apps with download numbers below this figure because they tend to choose the top mobile apps, which are ranked according to user comments and download count (28). The exclusion criteria were (1) duplicate and irrelevant apps (apps with the same name and producer were defined as duplicates, regardless of the availability of different versions); (2) apps without any meaningful introduction or instruction in the app store; (3) apps without a Chinese or English interface; (3) apps without a rating; (4) apps with fewer than 1 million downloads; (5) paid apps without trial; and (6) apps with no updates since January 1, 2020.

Two investigators independently chose the apps for inclusion in the study based on the inclusion criteria. The investigators extracted the following data from each included app: the app name, developer, specifications (medical, health, fitness, or unavailable), acquisition cost (free or in-app purchase), most recent update date, target users [women trying to conceive (TTC), pregnant women, postpartum women, those providing infant care, all types, or unspecified], safety statement (potential risks or "use under guidance" disclaimer), operating system (iOS or Android), supporting evidence (descriptive study, observational study, or randomized controlled trial), number of languages offered, user rating, and source of information (clinical guidelines). To avoid data omission, we compiled the aforementioned data into a spreadsheet, and each researcher used the same spreadsheet to document those data. Conflicts regarding inclusion and data extraction between the researchers were resolved through discussion.

\section{In-depth Analysis of Perinatal Care Apps}

To investigate the selected apps in depth, the apps were downloaded and installed on iOS and Android devices. Six independent researchers were each assigned the same number of apps. Two independent researchers registered and logged into each app to evaluate the quality of its content and functions; this ensured that each program was reviewed on both Apple App Store and the Android markets (Google, Huawei, Baidu, and 360). Where relevant, simulated input data, such as the predicted birth date and the start of the previous menstrual cycle, were utilized to accurately analyze the app's potential. For a comprehensive assessment of the functions of apps over the full pregnancy duration, two researchers pretended to be in the first trimester of pregnancy, two in the second trimester, and two in the third trimester. All investigators assessed and analyzed TTC and postpartum period information. The primary modules were recorded first, followed by the auxiliary functions inside each module after logging into the app. Each researcher utilized the same spreadsheet to document those data. Data on the apps' basic information of functionality and technological aspects were collected and analyzed between August 7 and October 20, 2021. The Fleiss Kappa value was used to measure the trustworthiness of the data gathered by the six researchers (33). The Mobile Application Rating Scale (MARS) was adopted to evaluate app quality through four dimensions of objective app quality, including engagement, functionality, aesthetics, and information quality (34). The subjective quality subscale of the MARS was omitted in the assessment since we aimed to assess the objective quality of apps. All MARS items were scored on a 5-point Likert scale ranging from 1 (inadequate) to 5 (excellent), with the researcher required to circle the number that most correctly indicated the quality of the app component under assessment (34). The mean scores for each dimension were calculated, and the mean total score of objective app quality was calculated across all 4 dimensions (34). Apps that scored $\geq 3$ out of 5 on the MARS were considered acceptable quality, and scores higher than 4 were rated as high quality (35). Three independent MARS-trained reviewers scored ratings to the apps. Disparities and uncertainties regarding the scores of apps among the researchers were addressed through discussion and reached a consensus on the final MARS scores. The component, runtime, and communication security of all apps were examined by the national anti-fraud center (version 1.1.17), which was established by China's Ministry of Public Security in 2021 (36). Some of its fundamental capabilities are anti-fraud early warning, identification verification, app self-examination, and risk inquiry, which effectively check the security performance of App and reduce the possibility of individuals being swindled (36). Scores $<60$ out of 100 on the test report represented a high risk, scores $\geq 60$ and $\leq 80$ represented a medium risk, and scores $>80$ represented low risk (36).

\section{Statistics Analyses}

The baseline features were summarized using Origin (2021) (Northampton, MA, USA), and a chi-square test or analysis of variance with a significance threshold of 0.05 was performed using OpenEpi (version 3.01) to compare the Chinese and US apps (37). The module's frequencies and percentages were calculated using Origin (2021) (Northampton, MA, USA). Further, a chi-square test with a significance threshold of 0.05 was performed using OpenEpi (version 3.01) to analyze the functional differences between the Chinese and US apps. 


\section{RESULTS}

\section{Basic Characteristics of the Included Apps}

Following a search of the Apple App Store, Google Play Store, Baidu Android Store, 360 Android Store, and Huawei Android Store, 817 apps were identified. After extensive screening, a total of 84 apps ( 39 from China and 45 from the US) were included. The flowchart in Figure 1 illustrates the app selection process.

The characteristics of all the included apps are summarized in Table 1. Among the Chinese mobile apps, $10.3 \%$ (4/39) were classified as medical, $79.4 \%$ (31/39) were classified as health and fitness, and $10.3 \%(4 / 39)$ were unclassified. A total of $89.7 \%$ $(35 / 39)$ of Chinese mobile apps lacked clear safety statements and supporting evidence, whereas among the US mobile apps, 53.3\% $(24 / 45)$ provided clear safety declarations, and $51.1 \%(23 / 45)$ supplied supporting evidence $(p<0.01)$. In terms of acquisition costs, the Chinese app markets offered more free apps than did the US ones [66.7\% (26/39) in China vs. $13.3 \%(6 / 45)$ in the US; $p$ $<0.01]$. Furthermore, a higher percentage of Chinese apps were targeted at women TTC and infant-care users compared with US apps [women TTC: $46.2 \%(18 / 39)$ in China vs. $13.3 \%(6 / 45)$ in the US; infant-care: $41 \%(16 / 39)$ in China vs. $2.2 \%(1 / 45)$ in the US; $p<0.05]$. In addition, Android systems were more common in China than in the US [79.5\% (31/39) in China vs. 31.1\% (14/45) in the US; $p<0.01]$. Multiple languages were available in $43.5 \%$ $(17 / 39)$ of Chinese apps, whereas multiple languages were only available for only $20 \%(9 / 45)$ of apps in the US $(p<0.01)$. In the US, none of the apps was uncategorized; $86.7 \%(39 / 45)$ of mobile apps were labeled as "health and fitness", whereas only $13.3 \%(6 / 45)$ were categorized as "medical". The number of inapp updates did not differ between the US and China apps after the COVID-19 outbreak; in 2021, 62.2\% (28/45) of US apps and $74.4 \%(29 / 39)$ of Chinese apps were updated. US apps had an average user rating of 3.41; this was slightly higher than that of the Chinese apps, which had a mean user rating of $3.09(p>0.05)$.

\section{Consistency of the Collected Content and Function Data of PtP Care Apps}

Six independent researchers were assigned the same number of apps and were tasked with collecting the apps' information of functionality and technical characteristics. The Fleiss Kappa value was 0.8403 , indicating that the authenticity and reliability of the apps' collected information of functions and technical characteristics were acceptable and could be analyzed.

\section{Functions and Modules}

The features of each function provided by the Chinese and US apps are represented in heat maps in Figures 2, 3, and the data are summarized in Table 2. Chinese and US PtP care mobile apps contained seven functions [monitoring (e.g., recording fetal size, tracking physical activity during pregnancy, water intake, recording baby photos), nutrition, general education, exercise, community, purchasing, and others] and were targeted at four applicable groups (women TTC, pregnant women, postpartum women, and those providing infant care). Fewer app functionalities were comparable between the two countries. The most common function of the 39 Chinese apps was fetal size monitoring $(n=18,46.2 \%)$, followed by general communication ( $n=16,41 \%)$, nutrition planning for pregnancy $(n=15,38.5 \%)$, and nutrition knowledge throughout pregnancy $(15,38.5 \%)$. The most common function of the 45 US mobile apps was pregnancy yoga ( $n=21,46.7 \%)$, followed by monitoring physical activity in pregnancy $(n=16,35.5 \%)$ and exercise during pregnancy ( $n=13,28.9 \%)$. Furthermore, none of the 39 Chinese mobile apps provided functions related to baby yoga, postpartum Pilates,

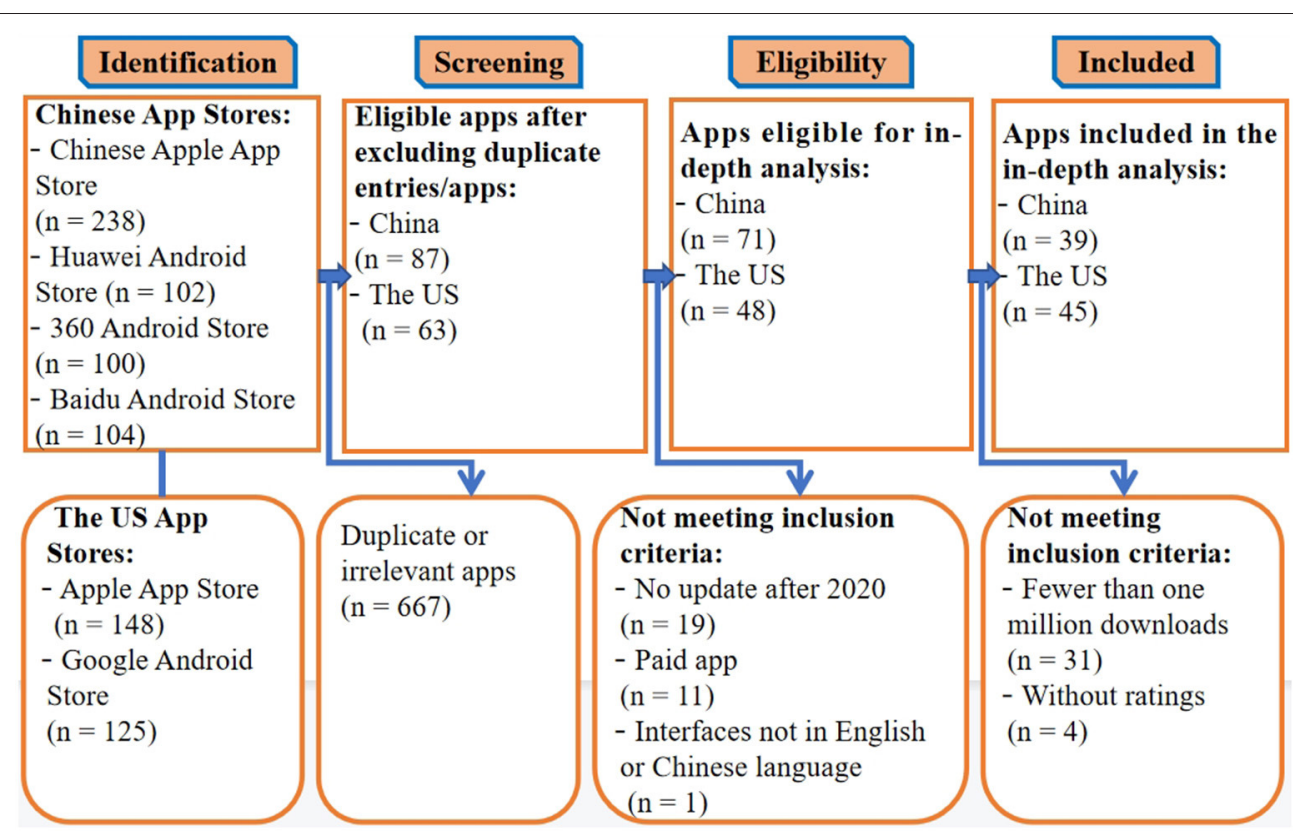

FIGURE 1 | Flowchart of the app selection process. 
TABLE 1 | Characteristics of the 84 apps for pregnancy to postpartum care identified in the US-China comparison.

\begin{tabular}{|c|c|c|c|c|}
\hline Category & China $(n=39)$ & $\begin{array}{l}\text { United States } \\
\qquad(n=45)\end{array}$ & $\chi^{2} / F$ & $p$ \\
\hline \multicolumn{5}{|l|}{ Specifications, $\boldsymbol{n}(\%)$} \\
\hline Medical & $4(10.3)$ & $6(13.3)$ & 1.158 & $p>0.05^{b}$ \\
\hline Health and fitness & $31(79.4)$ & $39(86.7)$ & & \\
\hline$N A^{d}$ & $4(10.3)$ & $0(0)$ & & \\
\hline \multicolumn{5}{|l|}{ Acquisition costs, $n(\%)$} \\
\hline Free & $26(66.7)$ & $6(13.3)$ & 25.200 & $p<0.01^{\star \star b}$ \\
\hline In-app purchase & $13(33.3)$ & $39(86.7)$ & & \\
\hline \multicolumn{5}{|c|}{ Target users (app description accompanying a clear statement), $n$ (\%) } \\
\hline Women TTC ${ }^{a}$ & $18(46.2)$ & $6(13.3)$ & 13.64 & $p<0.05^{\star b}$ \\
\hline Pregnant women & $28(71.8)$ & $32(71.1)$ & & \\
\hline Postpartum women & $16(41)$ & $23(51.1)$ & & \\
\hline Those providing infant care & $16(41)$ & $1(2.2)$ & & \\
\hline Not specified & $3(7.7)$ & $2(4.4)$ & & \\
\hline \multicolumn{5}{|l|}{ Safety statement, $\boldsymbol{n}(\%)$} \\
\hline With & $4(10.3)$ & $24(53.3)$ & 17.446 & $p<0.01^{* * b}$ \\
\hline Without & $35(89.7)$ & $21(46.7)$ & & \\
\hline \multicolumn{5}{|l|}{ Privacy policy, $n(\%)$} \\
\hline With & $35(89.7)$ & $42(93.3)$ & 0.039 & $p>0.05^{b}$ \\
\hline Without & $4(10.3)$ & $3(6.7)$ & & \\
\hline \multicolumn{5}{|l|}{ Supporting evidence, $\boldsymbol{n}(\%)$} \\
\hline With & $4(10.3)$ & $23(51.1)$ & 15.988 & $p<0.01^{\star \star b}$ \\
\hline Without & $35(89.7)$ & $22(48.9)$ & & \\
\hline \multicolumn{5}{|l|}{ Operating system, $n$ (\%) } \\
\hline iOS & $29(74.3)$ & $33(73.3)$ & 26.820 & $p<0.01^{\star * b}$ \\
\hline Android & $31(79.5)$ & $14(31.1)$ & & \\
\hline \multicolumn{5}{|c|}{ Year of the most recent update, $n(\%)$} \\
\hline 2020 & $10(25.6)$ & $17(37.8)$ & 1.411 & $p>0.05^{\mathrm{b}}$ \\
\hline 2021 & $29(74.4)$ & $28(62.2)$ & & \\
\hline \multicolumn{5}{|l|}{ Language, $\boldsymbol{n}(\%)$} \\
\hline Chinese & $22(56.4)$ & 0 & 63.562 & $p<0.01^{\star * b}$ \\
\hline English & 0 & $36(80)$ & & \\
\hline Multiple languages offered & $17(43.6)$ & $9(20)$ & & \\
\hline Mean user rating (stars/5) & $3.09 \pm 0.70$ & $3.41 \pm 0.9$ & 3.198 & $p>0.05^{c}$ \\
\hline
\end{tabular}

a Trying to conceive.

${ }^{b}$ Chi-square test.

${ }^{c}$ Analysis of variance.

${ }^{d}$ Not available.

${ }^{* *}$ Extremely significant difference at $p<0.01$

* Significant difference at $p<0.05$.

meditation, treatment for psychosocial concerns, breathing exercises, postpartum pelvic exercises, pregnancy Pilates, or pelvic exercise. The following features were not included in any of the 45 US mobile apps: recording baby photos, pregnancy check-up reminders, recording contractions, recording ovulation date, infant vaccination reminders, recording pregnancy photos, recording temperature, ovulation reminders, recording the baby's weight, or addressing psychosocial concerns.

Our quantitative analysis revealed the following significant differences between China and the US in terms of the functions offered by the PtP care apps. (1) Monitoring: recording fetal size
( $n=18,46.2 \%$ in China vs. $n=3,6.7 \%$ in the US) and tracking physical activity during pregnancy $(n=6,15.4 \%$ in China vs. $n$ $=16,35.5 \%$ in the US); (2) nutrition: nutrition planning during pregnancy ( $n=15,38.5 \%$ in China vs. $n=7,15.6 \%$ in the US); (3) general education: nutrition knowledge during pregnancy ( $n$ $=15,38.5 \%$ in the China vs. $n=5,11.1 \%$ in the US); (4) exercise: pregnancy yoga $(n=2,5.1 \%$ in China vs. $n=21,46.7 \%$ in the US), pregnancy workouts ( $n=2,5.1 \%$ in China vs. $n=13,28.9 \%$ in the US), and pregnancy meditation ( $n=0,0 \%$ in China vs. $n$ $=10,22.2 \%$ in the US); (5) community: general communication ( $n=16,41 \%$ in China vs. $n=6,13.3 \%$ in the US) and patientclinician communication ( $n=14,35.9 \%$ in the China vs. $n=0$, $0 \%)$; (6) shopping: pregnancy and baby product sales $(n=11$, $28.2 \%$ in China vs. $n=2,4.4 \%$ in the US), (7) others: baby stories ( $n=10,25.6 \%$ in the China vs. $n=0,0 \%$ in the US) and baby music ( $n=7,17.9 \%$ in the China vs. $n=3,6.7 \%$ in the US).

\section{App Quality Based on the MARS}

The overall MARS scores for app quality ranged from a minimum score of 2.7 to a maximum of 4.4 (median 3.5) in the US vs. 2.3 to 4.2 (median 3.2) in China, with most apps (34/45, 76\% in the US vs. $23 / 39,59 \%$ in China) achieving a score $>3$. The engagement score ranged from 2.2 to 4.4 (median 3.6) in the US vs. 1.2 to 4.4 (median 2.9) in the China. The functionality score ranged from 3.0 to 4.8 (median 4.0 ) in the US vs. 2.5 to 4.5 (median 3.7 ) in the China. The esthetics score ranged from 2.3 to 4.4 (median 3.8 ) in the US vs. 2.3 to 4.7 (median 3.6 ) in the China, and the information score ranged from 1.2 to 4.9 (median 2.9) in the US vs. 1.2 to 4.2 (median 2.4) in the China. The mean MARS scores for all 84 analyzed Chinese and US apps are reported in Supplementary Table 1.

\section{Risk Assessment of PtP Care Apps in the US and China}

The security risks associated with the 84 apps were assessed using the anti-fraud software. Among US mobile apps, 8.9 and $40 \%$ were considered high- and medium-risk, respectively; among Chinese apps, 7.6\% (3/39) were deemed to be highrisk (Table 3 ). The risk of PtP care apps differed significantly between the US and China ( $p<0.01$; Table 3). According to the risk assessment report, "high-risk" generally suggests that the program includes hazardous information or third-party plugins, whereas "medium-risk" implies the possibility of personal information leakage.

\section{DISCUSSION}

This study systematically demonstrated the features and functionalities of in-store mobile apps for PtP care in the US and China, two of the largest app marketplaces, offering an overview of their primary characteristics and functions with a special emphasis on weaknesses and gaps to be addressed with future eHealth-related innovations. We believe that such an investigation is essential for developing more effective PtP health apps during COVID-19. Pregnancy and postnatal education and support provided by a multidisciplinary team of specialists may 


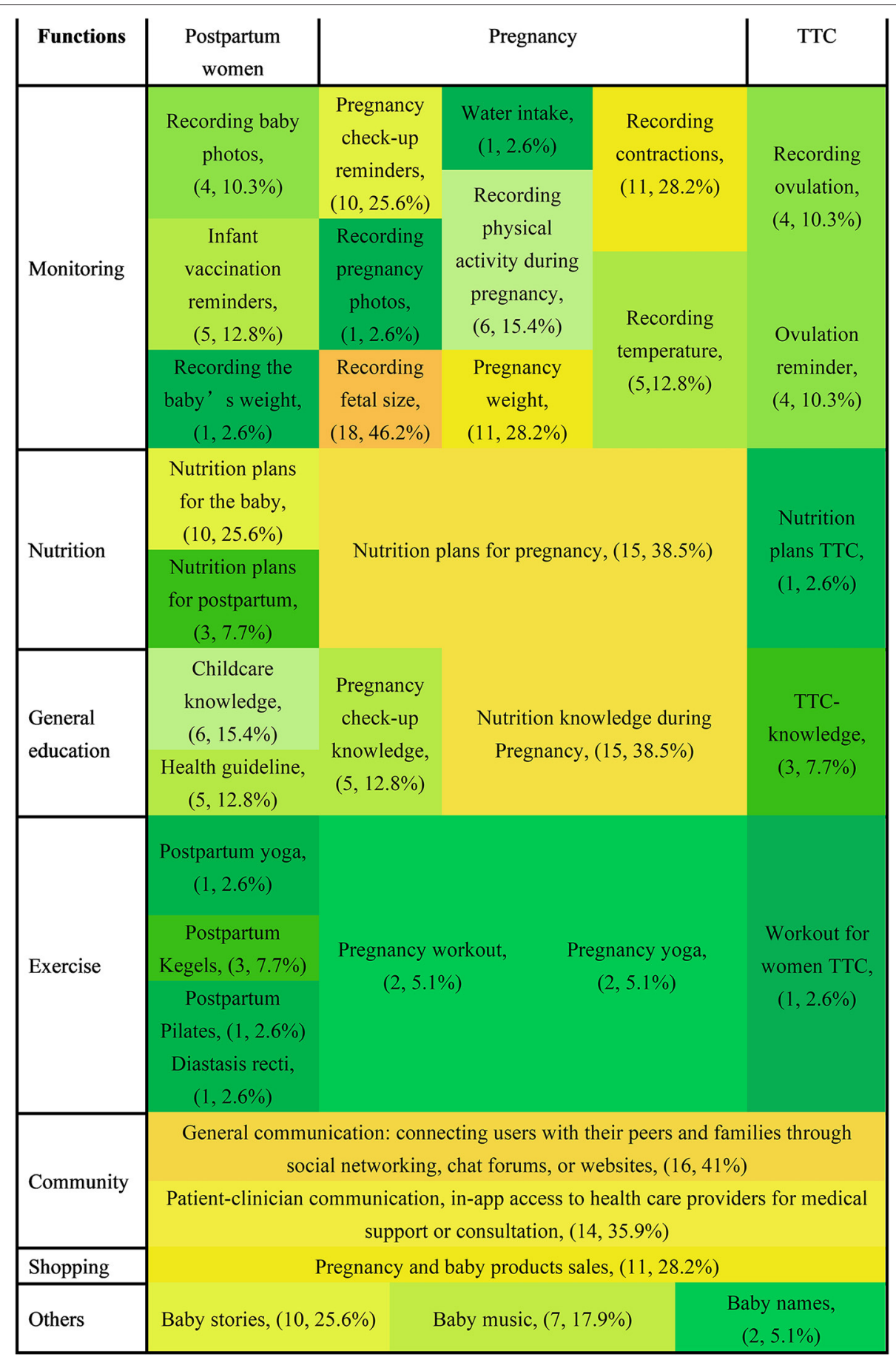

FIGURE 2 | Heat map of features of the 39 Chinese mobile apps for pregnancy to postnatal care. TTC, trying to conceive.

improve the quality of care through apps, even among lowincome groups or groups in impoverished areas. Overall, our study reveals that (1) there are differences in PtP care mobile apps between the two countries; (2) the quality and effectiveness of the information offered by apps must be carefully examined and combined with professional medical advice; and (3) some Chinese and US apps were suspected of stealing users' personal information. These findings do not support our hypothesis and highlight key areas for improvement of apps targeted at pregnant and postpartum women. 


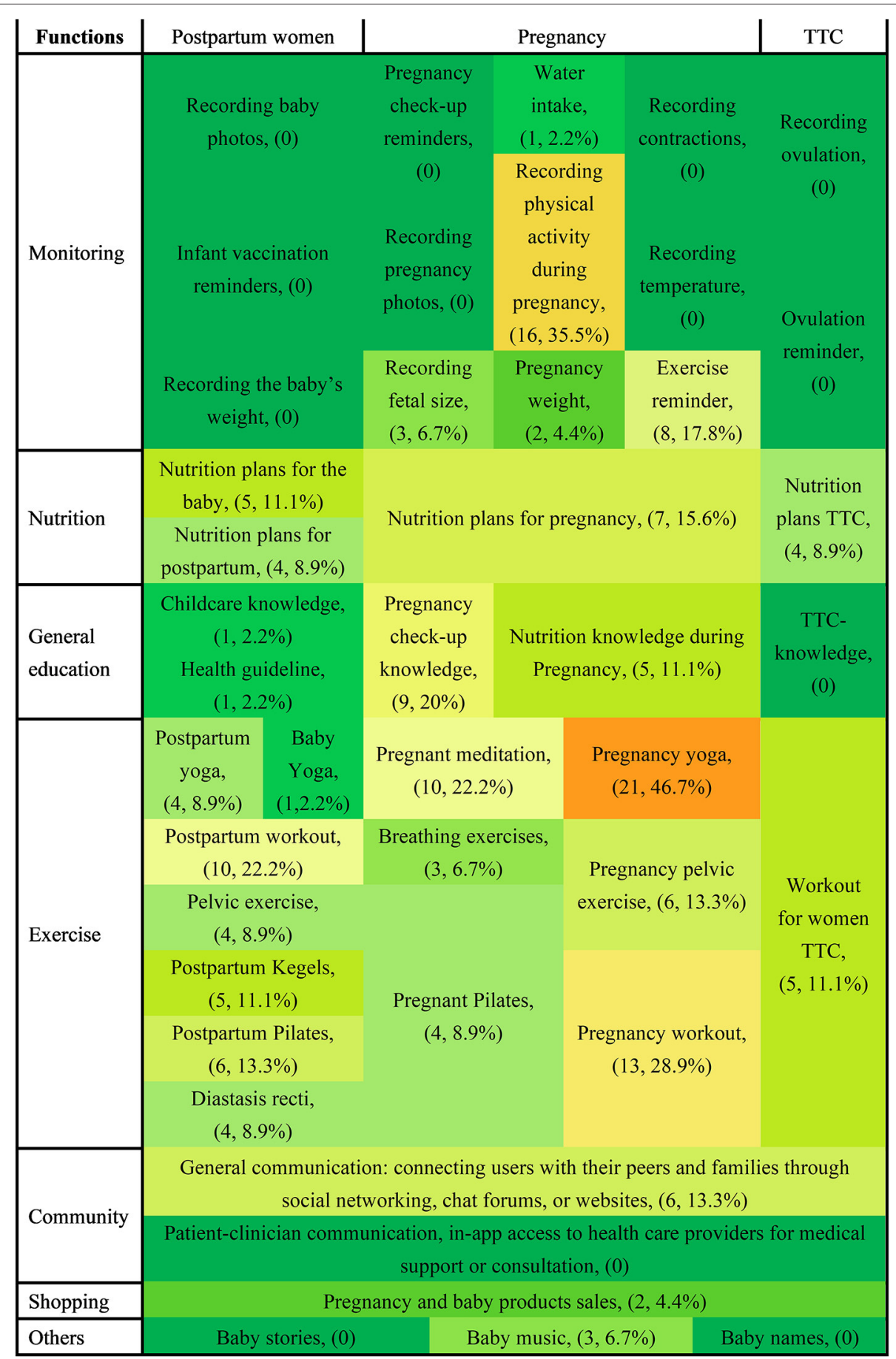

FIGURE 3 | Heat map of features of the 45 US mobile apps for pregnancy and postnatal care. TTC, trying to conceive.

Overview of Hypothesis Validation Findings Metadata of Apps

All 45 US apps were classified as medical or health and fitness apps, but several Chinese apps $(10.3 \%, 4 / 39)$ were uncategorized. This may be because the Food and Drug Administration (FDA) risk studies and policy recommendations for mobile health technology require apps to be categorized into three categories: health management, general management, and medical devices (38). Another possible explanation is that the FDA regulates all mobile health technology (38). Unlike the US, China lacks any such legislation. Additionally, few apps were classified as medical devices, indicating a severe weakness in Chinese-US 
TABLE 2 | Comparison of the characteristics of the mobile apps for pregnancy and postnatal care between the US and China.

\begin{tabular}{|c|c|c|c|c|}
\hline Category & China $(n=39)$ & United States $(n=45)$ & $\chi^{2}$ & $p$ \\
\hline \multicolumn{5}{|l|}{ Log, $n(\%)$} \\
\hline Recording baby photos & $4(10.3)$ & $0(0)$ & & \\
\hline Recording the baby's weight & $1(2.6)$ & $0(0)$ & & \\
\hline Pregnancy check-up reminders & $10(25.6)$ & $0(0)$ & & \\
\hline Water intake & $1(2.6)$ & $1(2.2)$ & & \\
\hline Recording physical activity during pregnancy & $6(15.4)$ & $16(35.5)$ & & \\
\hline Exercise reminder & $0(0)$ & $8(17.8)$ & & \\
\hline Pregnancy weight & $11(28.2)$ & $2(4.4)$ & & \\
\hline Recording contractions & $11(28.2)$ & $0(0)$ & & \\
\hline Nutrition plans for baby & $10(25.6)$ & $5(11.1)$ & & \\
\hline Nutrition plans for postpartum women & $3(7.7)$ & $4(8.9)$ & 25.200 & $p<0.01^{\star *}$ \\
\hline Nutrition plans for pregnant women & $15(38.5)$ & $7(15.6)$ & & \\
\hline Nutrition plans for women $T T \mathrm{C}^{\mathrm{a}}$ & $1(2.6)$ & $4(8.9)$ & & \\
\hline \multicolumn{5}{|l|}{ General education, $n(\%)$} \\
\hline Childcare knowledge & $6(15.4)$ & $1(2.2)$ & & \\
\hline Health guideline & $5(12.8)$ & $1(2.2)$ & 91.13 & $p<0.01^{\star *}$ \\
\hline Pregnancy check-up knowledge & $5(12.8)$ & $9(20)$ & & \\
\hline Nutrition knowledge during pregnancy & 15 (38.5) & $5(11.1)$ & & \\
\hline TTC knowledge & $3(7.7)$ & $0(0)$ & & \\
\hline Pregnancy meditation & $0(0)$ & $10(22.2)$ & & \\
\hline Pregnancy yoga & $2(5.1)$ & $21(46.7)$ & & \\
\hline Pregnancy pelvic exercise & $0(0)$ & $6(13.3)$ & & \\
\hline Pregnancy workout & $2(5.1)$ & $13(28.9)$ & & \\
\hline Pregnancy Pilates & $0(0)$ & $4(8.9)$ & & \\
\hline Workout for women TTC & $1(2.6)$ & $5(11.1)$ & & \\
\hline \multicolumn{5}{|l|}{ Community, $n$ (\%) } \\
\hline General communication & $16(41)$ & $6(13.3)$ & & \\
\hline Patient-clinician communication & $14(35.9)$ & $0(0)$ & 8.288 & $p<0.01^{* *}$ \\
\hline \multicolumn{5}{|l|}{ Shopping, $n$ (\%) } \\
\hline With shopping function or service & $11(28.2)$ & $2(4.4)$ & 9.017 & $p<0.01^{\star *}$ \\
\hline \multicolumn{5}{|l|}{ Others, $n(\%)$} \\
\hline Baby stories & $10(25.6)$ & $0(0)$ & & \\
\hline Baby music & $7(17.9)$ & $3(6.7)$ & 35.122 & $p<0.01^{\star *}$ \\
\hline Baby names & $2(5.1)$ & $0(0)$ & & \\
\hline
\end{tabular}

${ }^{a}$ Trying to conceive.

${ }^{* *}$ Extremely significant difference at $p<0.01$. 
apps. To overcome this issue, apps must not only be designed as information or entertainment tools but also be subjected to new medical device legislation. This legislation emphasizes the importance of anticipating the use of medical device apps by mothers and the role of these apps in controlling or assisting conception $(39,40)$. Furthermore, each Chinese app covered a broader range of target users than US apps. Three Chinese apps and two US apps did not specify their target users. By contrast, a few more US apps noted that it was vital for app developers to differentiate their products to match the specific demands of diverse populations (41). A previous study reported that pregnant and postpartum women in China had considerably different demands than did women in the US (5), which may explain the difference in target users.

Regarding supporting evidence and safety statements, only $10.3 \%$ of the Chinese apps offered references to the information provided and recognized their scientific responsibility. The mean value of the information was lower than the acceptable level in both the US and China. Pregnant and postpartum women may be more vulnerable to inaccurate information (26). Given the potential of mHealth apps to promote maternal health and knowledge about pregnancy and children's health and development, apps capable of providing the most comprehensive, accurate, and trustworthy information on pregnancy and the postnatal period are urgently needed.

Regarding acquisition costs, $\sim 80 \%$ of US apps offered inapp purchases; this was connected to the difference in the exercise functions, as American apps offered plentiful training courses for pregnancy and exercise-related postpartum recovery. Exercise during pregnancy is increasingly popular in the US $(42,43)$, which may explain the large disparity in acquisition costs between the two countries. The addition of the fitness element in US apps is a worthwhile endeavor, particularly amid the current COVID-19 outbreak. Effective online prenatal exercise classes are useful to both mothers and their unborn children (11). Noticeable distinctions were observed between Chinese and US apps in terms of the operating system and language. Chinese apps tend to offer multiple languages and favor Android operating systems compared to their US counterparts. This may be related to China's increasing mobile market share in domestic and foreign markets in recent years (44).

User feedback is a true reflection of users' requirements and genuine experiences using the app $(45,46)$. Objective app quality reflects the app's information, functionality, aesthetics, and engagement (47). Both countries' user and objective app quality ratings were at acceptable levels, with the US marginally outperforming China in these categories and the engagement score of quality being poor in China. The development of PtP apps may be improved if the apps are highly valued and used by the target audience (46). The abovementioned results did not support our hypothesis that the most updated content offered by the app would be high-quality and effective. This suggests that the features of apps available in both the US and China should be improved, and more interaction between app developers and users is recommended.
TABLE 3 | The risk assessment of pregnancy and postnatal care mobile apps in the US and China.

\begin{tabular}{lccc}
\hline Risk & $\begin{array}{c}\text { United States } \\
(\boldsymbol{n}=\mathbf{4 5}), \boldsymbol{n} \\
(\mathbf{\%})\end{array}$ & China $(\boldsymbol{n}=\mathbf{3 9 )}, \boldsymbol{n}(\mathbf{\%})$ & $\boldsymbol{p}$-value \\
\hline Low & $23(51.1)$ & $4(10.3)$ & \\
Medium & $18(40)$ & $32(82.1)$ & $p<0.01^{\text {** }}$ \\
High & $4(8.9)$ & $3(7.6)$ & $p<0.01^{\text {** }}$ \\
\hline
\end{tabular}

${ }^{* \star}$ Extremely significant difference at $p<0.01$.

\section{Functions and Modules}

According to our data, monitoring, nutrition, general education, exercise, community, shopping, and other features were the most utilized functions in prenatal care mobile apps in both China and the US. However, psychological services were seldom observed in either country's apps, even though psychological support is strongly related to pregnant and postpartum women's mental health (48). The primary impediment to developing this function and module may be its complicated nature (49).

Exercise-related features (e.g., pregnancy yoga, fitness, and meditation) were more frequent in US-based apps than in Chinese-based apps. We have already discussed the reasons for the discrepancies in the exercise modules during the pregnancy and postpartum periods. Research has demonstrated that exercise is beneficial during pregnancy (50), but because of a lack of funds and expert advice, many pregnant women do not achieve the recommended amount of physical activity $(51,52)$. Therefore, we propose that the Chinese apps should be upgraded to incorporate exercise modules to effectively address the aforementioned issues because online courses are less expensive than courses offered at professional organizations. This ensures that even in impoverished regions and during the COVID-19 lockdown, users can access relevant exercise modules (53).

Monitoring functionalities (e.g., tracking fetal size, physical activity during pregnancy, and contractions) were more common in Chinese apps than in US apps. These services are based on built-in algorithms (generally predictive modeling utilizing acquired personal data and possibly sophisticated methods, such as artificial intelligence) and provide users with immediate and direct advice depending on their circumstances (54). These functionalities play a crucial role, as offline healthcare may be unavailable during the COVID-19 pandemic (55). However, monitoring modules should be constructed with care and prudence, and further attempts with alternative algorithms should be encouraged simultaneously. The study findings also revealed that none of the apps directly enabled mothers to schedule appointments for medical treatment, immunization, or physical examinations, highlighting the absence of a direct link between apps and appointment scheduling systems. We recommend that $\mathrm{PtP}$ care apps be connected to a wider regional or national public health care network. This substantial gap could be solved by developing more networked and institutional apps to improve booking processes by lowering the number of calls or other request types from patients and the professionals that manage them (56). 


\section{Risk Assessment}

Risk assessment is primarily concerned with two factors: the content and the app program. The content risk evaluation was primarily based on information quality, security statements, and scientific evidence. According to the findings of the information quality, supporting scientific evidence, and safety statement inquiries, the content-related risk was mostly due to a lack of safety statements and published scientific literature or had positive outcomes in studies that are not RCTs. This issue was more prominent in Chinese apps than in US apps. The app program checks revealed that several of these apps required the user to provide personal information beyond the extent of their permissions. The app self-examination by the national antifraud center measured the security of the installation package and program usage. The report demonstrated that certain apps compelled users to provide permission, claimed too many privileges, and gathered personal information that was outside of their scope. Analysts noted that mobile apps have a strong competitive edge in the market; apps would be unlikely to work if users do not accept the privacy policies (57). The above information does not support our hypothesis that all apps would be concerned with users' personal information and data privacy and security. We propose that users carefully read the privacy statements and that government agencies improve internet surveillance.

\section{Strengths and Limitations}

Several benefits and drawbacks of the present study should be considered when evaluating the conclusions. To the best of our knowledge, this study is the first to evaluate the quality and risks associated with in-store PtP care apps in China and the US. Second, we acted as users at various PtP stages to download and utilize the apps, which may or may not have resulted in overlooking functional information about the mobile apps. Third, the consistency and dependability of the data gathered were rigorously evaluated before being used. Finally, apps available in two countries with distinct cultures, healthcare systems $(58,59)$, and economic standings were analyzed. This study also has several drawbacks. First, we identified apps at a single time point, which may have omitted longitudinal updates to app features. Second, given the nature of the characteristics under investigation based on the user ratings, subjectivity in assigning ratings cannot be ruled out. Third, we excluded paid apps, which may have omitted some available information. Finally, we did not include apps from other countries because of linguistic barriers. Future researchers interested in this topic may wish to investigate different time periods to increase the reliability of longitudinal comparisons on functionality and technological aspects in more countries. Additionally, we will further analyze the influence of PtP apps on PtP health care.

\section{REFERENCES}

1. Asselmann E, Kunas SL, Wittchen H-U, Martini J. Maternal personality, social support, and changes in depressive, anxiety, and stress symptoms during pregnancy and after delivery: a prospective-longitudinal study. PLOS ONE. (2020) 15:1-18. doi: 10.1371/journal.pone.0237609

\section{CONCLUSIONS}

In summary, the in-store mobile PtP care apps differed between China and the US regarding their functionality and characteristics. Both countries' apps, and particularly Chinese apps, have their share of issues; these include lack of evidencebased information, risk of inaccurate content, and lack of app evaluation. Monitoring-related information was more common in Chinese apps, whereas exercise-related content was more abundant in US apps. Both countries' apps also failed to provide adequate mental health services. These findings highlight the need for improving the features of PtP care apps available in both countries, and extensive interaction between app developers and users is suggested. Maintaining a suitable level of regulation is necessary to ensure the quality and functionality of in-store apps. Basic public health services for women's health may improve with the development of high-efficiency PtP apps.

\section{DATA AVAILABILITY STATEMENT}

The original contributions presented in the study are included in the article/Supplementary Material, further inquiries can be directed to the corresponding author.

\section{AUTHOR CONTRIBUTIONS}

HY: conceptualization, funding acquisition, software, validation, visualization, and writing-original draft. HY, JH, XW, WY, and BS: data curation and formal analysis. $\mathrm{HY}$ and $\mathrm{JH}$ : methodology. HY and AS: project administration, resources, supervision, writing-review, and editing. All authors revised and approved the manuscript.

\section{FUNDING}

Gdansk University of Physical Education and Sport supported this research.

\section{ACKNOWLEDGMENTS}

We would like to thank Dr. Arif Muhammad, senior researcher, for his methodological and technical support in the whole research process.

\section{SUPPLEMENTARY MATERIAL}

The Supplementary Material for this article can be found online at: https://www.frontiersin.org/articles/10.3389/fpubh. 2022.826896/full\#supplementary-material

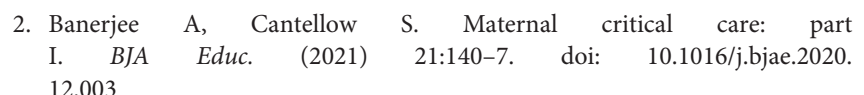

. Jingmei M, Huixia Y. Proceedings of the $22^{\text {nd }}$ World Congress of the International Federation of Gynecology and Obstetrics (Perinatal Medicine). J Perinat Med. (2018) 21:2. doi: 10.3760/cma.j.issn.1007-9408.2018 
4. Thayer ZM, Rutherford J, Kuzawa CW. The maternal nutritional buffering model: an evolutionary framework for pregnancy nutritional intervention. Evol Med Public Health. (2020) 2020:14-27. doi: 10.1093/emph/eoz037

5. Withers M, Kharazmi N, Lim E. Traditional beliefs and practices in pregnancy, childbirth and postpartum: a review of the evidence from Asian Countries. Midwifery. (2018) 56:158-70. doi: 10.1016/j.midw.2017.10.019

6. Lomax A. Examination of the newborn: professional issues in practice. In: Anne L, editor. Examination of the Newborn: An Evidence-Based Guide. Oxford, UK: Nursing Standard. (2021). p. 211-6.

7. Atif N, Nazir H, Zafar S, Chaudhri R, Atiq M, Mullany LC, et al. Development of a psychological intervention to address anxiety during pregnancy in a low-income country. Front Psychiatry. (2020) 10:927. doi: 10.3389/fpsyt.2019.00927

8. Poon Z, Lee ECW, Ang LP, Tan NC. Experiences of primary care physicians managing postpartum care: a qualitative research study. BMC Fam Pract. (2021) 22:1-12. doi: 10.1186/s12875-021-01494-w

9. Callander EJ, Gamble J, Creedy DK. Postnatal major depressive disorder in Australia: inequalities and costs of healthcare to individuals, governments and insurers. Pharmacoeconomics. (2021) 39:731-9. doi: 10.1007/s40273-021-01013-w

10. Vasco M, Pandya S, Van Dyk D, Bishop DG, Wise R, Dyer RA. Maternal critical care in resource-limited settings. Narrative review. Int J Obstet Anesth. (2019) 37:86-95. doi: 10.1016/j.ijoa.2018.09.010

11. Hessami K, Romanelli C, Chiurazzi M, Cozzolino M. COVID-19 pandemic and maternal mental health: a systematic review and meta-analysis. J MaternFetal Neonatal Med. (2020) 2020:1-8. doi: 10.1080/14767058.2020.1843155

12. Iyawa GE, Hamunyela S. mHealth apps and services for maternal healthcare in developing countries. In: 2019 IST-Africa Week Conference [Conference Presentation]. Nairobi: IEEE (2019). Available online at: https://ieeexplore. ieee.org/abstract/document/8764878 (accessed July 18, 2019).

13. Murthy P, Naji M. Role of digital health, mhealth, and low-cost technologies in advancing universal health coverage in emerging economies. In: Padmini M,editor. Technology and Global Public Health. Berlin: Springer (2020). p. 3146.

14. Frid G, Bogaert K, Chen KT. Mobile health apps for pregnant women: systematic search, evaluation, and analysis of features. J Medical Internet Res. (2021) 23:e25667. doi: 10.2196/25667

15. Jaks R, Baumann I, Juvalta S, Dratva J. Parental digital health information seeking behavior in Switzerland: a cross-sectional study. BMC Public Health. (2019) 19:225. doi: 10.1186/s12889-0196524-8

16. Sherifali D, Nerenberg KA, Wilson S, Semeniuk K, Ali MU, Redman LM, et al. The effectiveness of ehealth technologies on weight management in pregnant and postpartum women: systematic review and meta-analysis. J Med Internet Res. (2017) 19:1. doi: 10.2196/jmir.8006

17. Chan KL, Chen M. Effects of social media and mobile health apps on pregnancy care: meta-analysis. JMIR mHealth uHealth. (2019) 7:e11836. doi: 10.2196/11836

18. Tinius RA, Polston M, Bradshaw H, Ashley P, Greene A, Parker AN. An assessment of mobile applications designed to address physical activity during pregnancy and postpartum. Int J Exerc Sci. (2021) 14:382-99.

19. Hayman M, Alfrey K-L, Cannon S, Alley S, Rebar AL, Williams S, et al. Quality, features, and presence of behavior change techniques in mobile apps designed to improve physical activity in pregnant women: systematic search and content analysis. JMIR mHealth uHealth. (2021) 9:e23649. doi: 10.2196/23649

20. Lau Y, Cheng J-Y, Wong S-H, Yen K-Y, Cheng L-J. Effectiveness of digital psychotherapeutic intervention among perinatal women: a systematic review and meta-analysis of randomized controlled trials. World J Psychiatry. (2021) 11:133-52. doi: 10.5498/wjp.v11.i4.133

21. Qian J, Wu T, Lv M, Fang Z, Chen M, Zeng Z, et al. The value of mobile health in improving breastfeeding outcomes among perinatal or postpartum women: systematic review and meta-analysis of randomized controlled trials. JMIR Mhealth Uhealth. (2021) 9:e26098. doi: 10.2196/26098

22. Daly LM, Horey D, Middleton PF, Boyle FM, Flenady V. The effect of mobile app interventions on influencing healthy maternal behavior and improving perinatal health outcomes: systematic review. JMIR Mhealth Uhealth. (2018) 6:e10012. doi: $10.2196 / 10012$
23. Brown HM, Bucher T, Collins CE, Rollo ME. A review of pregnancy iphone apps assessing their quality, inclusion of behaviour change techniques, and nutrition information. Matern Child Nutr. (2019) 15:e12768. doi: 10.1111/mcn.12768

24. Sardi L, Idri A, Redman LM, Alami H, Bezad R, Fernández-Alemán JL. Mobile health applications for postnatal care: review and analysis of functionalities and technical features. Comput Methods Programs Biomed. (2020) 184:105114. doi: 10.1016/j.cmpb.2019.105114

25. Brunelli L, De Vita C, Cenedese F, Cinello M, Paris M, Samogizio F et al. Gaps and future challenges of Italian apps for pregnancy and postnatal care: systematic search on app stores. J Med Internet Res. (2021) 23:e29151. doi: 10.2196/29151

26. Overdijkink SB, Velu AV, Rosman AN, van Beukering MD, Kok M, Steegers-Theunissen RP. The usability and effectiveness of mobile health technology-based lifestyle and medical intervention apps supporting health care during pregnancy: systematic review. JMIR mHealth uHealth. (2018) 6:e109. doi: 10.2196/mhealth.8834

27. Taouk L, Schulkin J, Gunthert K. Brief report: the moderating effect of stress mindsets on associations between stress during pregnancy and symptoms of depression and anxiety. Anxiety Stress Coping. (2021) 2021:110. doi: 10.1080/10615806.2021.1967937

28. Biviji R, Vest JR, Dixon BE, Cullen T, Harle CA. Factors related to user ratings and user downloads of mobile apps for maternal and infant health: crosssectional study. JMIR mHealth uHealth. (2020) 8:e15663. doi: 10.2196/15663

29. Chen Q, Carbone ET. Functionality, implementation, impact, and the role of health literacy in mobile phone apps for gestational diabetes: scoping review. JMIR Diabetes. (2017) 2:e25. doi: 10.2196/diabetes.8045

30. Taki S, Russell CG, Lymer S, Laws R, Campbell K, Appleton J, et al. A mixed methods study to explore the effects of program design elements and participant characteristics on parents' engagement with an mHealth program to promote healthy infant feeding: the growing healthy program. Front Endocrinol. (2019) 10:397. doi: 10.3389/fendo.2019.00397

31. Balapour A, Nikkhah HR, Sabherwal R. Mobile application security: role of perceived privacy as the predictor of security perceptions. Int J Inf Manage. (2020) 52:102063. doi: 10.1016/j.ijinfomgt.2019.102063

32. Criss S, Woo Baidal JA, Goldman RE, Perkins M, Cunningham C, Taveras EM. The role of health information sources in decision-making among hispanic mothers during their children's first 1000 days of life. Matern Child Health J. (2015) 19:2536-43. doi: 10.1007/s10995-015-1774-2

33. Rigby AS. Statistical methods in epidemiology. V. towards an understanding of the kappa coefficient. Disabil Rehabil. (2000) 22:339-44. doi: 10.1080/096382800296575

34. Stoyanov SR, Hides L, Kavanagh DJ, Zelenko O, Tjondronegoro D, Mani M. Mobile app rating scale: a new tool for assessing the quality of health mobile apps. JMIR mHealth uHealth. (2015) 3:e27. doi: 10.2196/mhealth.3422

35. Stec MA, Arbour MW, Hines HF. Client-centered mobile health care applications: using the mobile application rating scale instrument for evidence-based evaluation. J Midwif Womens Health. (2019) 64:3249. doi: 10.1111/jmwh.12941

36. The Ministry of Public Security of the People's Republic of China. National Anti-Fraud Center Official Number Online. (2021). Available online at: https:/www.mps.gov.cn/n2254098/n4904352/c7711985/content. html (accessed February 2, 2021).

37. Shen C, Panda S, Vogelstein JT. The chi-square test of distance correlation. J Comput Graph Stat. (2021) 2021:1-15. doi: 10.1080/10618600.2021.1938585

38. Cortez NG, Cohen IG, Kesselheim AS. FDA regulation of mobile health technologies. N Engl J Med. (2014) 371:372-9. doi: 10.1056/NEJMhle14 03384

39. Agu E, Pedersen P, Strong D, Tulu B, He Q, Wang L, et al. The smartphone as a medical device: assessing enablers, benefits and challenges [Conference Presentation].In: 2013 IEEE International Workshop of Internet-of-Things Networking and Control (IoT-NC). Los Angeles, CA (2013). Available onlnie at: https://ieeexplore.ieee.org/abstract/document/6694053 (accessed January $6,2014)$.

40. Albrecht U-V, Malinka C, Long S, Raupach T, Hasenfuß G, von Jan U. Quality principles of app description texts and their significance in deciding to use health apps as assessed by medical students: survey study. JMIR mHealth uHealth. (2019) 7:e13375. doi: 10.2196/13375 
41. Zhou L, Bao J, Setiawan IMA, Saptono A, Parmanto B. The mHealth App Usability Questionnaire (MAUQ): development and validation study. JMIR mHealth uHealth. (2019) 7:e11500. doi: 10.2196/11500

42. Evenson KR, Savitz DA, Huston SL. Leisure-time physical activity among pregnant women in the US. Paediatr Perinat Epidemiol. (2004) 18:4007. doi: 10.1111/j.1365-3016.2004.00595.x

43. Evenson KR, Wen F. National trends in self-reported physical activity and sedentary behaviors among pregnant women: NHANES 1999-2006. Prev Med. (2010) 50:123-8. doi: 10.1016/j.ypmed.2009.12.015

44. Li D, Capone G, Malerba F. The long march to catch-up: a history-friendly model of China's Mobile Communications Industry. Res Policy. (2019) 48:649-64. doi: 10.1016/j.respol.2018.10.019

45. Pagano D, Maalej W. User feedback in the appstore: an empirical study [Conference Presentation]. In: 2013 21st IEEE International Requirements Engineering Conference (RE). Rio de Janeiro (2013). Available online at: https://ieeexplore.ieee.org/abstract/document/6636712 (accessed October 21, 2013).

46. Guzman E, Oliveira L, Steiner Y, Wagner LC, Glinz M. User feedback in the app store: a cross-cultural study [Conference Presentation]. In: 2018 IEEE/ACM 40th International Conference on Software Engineering: Software Engineering in Society (ICSE-SEIS). Gothenburg (2018). Available online at: https://ieeexplore.ieee.org/abstract/document/8445154 (accessed June 3, 2018).

47. Agarwal S, LeFevre AE, Lee J, L'Engle K, Mehl G, Sinha C, et al. Guidelines for reporting of health interventions using mobile phones: mobile health (mHealth) evidence reporting and assessment (mERA) checklist. BMJ. (2016) 352:i1174. doi: 10.1136/bmj.i1174

48. Basu A, Kim HH, Basaldua R, Choi KW, Charron L, Kelsall N, et al. A cross-national study of factors associated with women's perinatal mental health and wellbeing during the COVID-19 pandemic. PLoS ONE. (2021) 16:1-18. doi: 10.1371/journal.pone.0249780

49. Bjelica A, Cetkovic N, Trninic-Pjevic A, Mladenovic-Segedi L. The phenomenon of pregnancy - a psychological view. Ginekol Pol. (2018) 89:1026. doi: 10.5603/GP.a2018.0017

50. Tsakiridis I, Bakaloudi DR, Oikonomidou AC, Dagklis T, Chourdakis M. Exercise during pregnancy: a comparative review of guidelines. J Perinat Med. (2020) 48:519-25. doi: 10.1515/jpm-2019-0419

51. Harrison AL, Taylor NF, Shields N, Frawley HC. Attitudes, barriers and enablers to physical activity in pregnant women: a systematic review. J Physiother. (2018) 64:24-32. doi: 10.1016/ j.jphys.2017.11.012

52. Walasik I, Kwiatkowska K, Kosińska Kaczyńska K, Szymusik I. Physical activity patterns among 9000 pregnant women in Poland: a cross-sectional study. Int J Environ Res Public Health. (2020) 17:1771. doi: 10.3390/ijerph17051771
53. Mutz M, Müller J, Reimers AK. Use of digital media for homebased sports activities during the COVID-19 pandemic: results from the German SPOVID Survey. Int J Environ Res Public Health. (2021) 18:4409. doi: 10.3390/ijerph18094409

54. Baxter C, Carroll J-A, Keogh B, Vandelanotte C. Assessment of mobile health apps using built-in smartphone sensors for diagnosis and treatment: systematic survey of apps listed in international curated health app libraries. JMIR mHealth uHealth. (2020) 8:e16741. doi: 10.2196/16741

55. Kapoor A, Guha S, Kanti Das M, Goswami KC, Yadav R. Digital healthcare: the only solution for better healthcare during COVID-19 pandemic? Indian Heart J. (2020) 72:61-4. doi: 10.1016/j.ihj.2020.04.001

56. Lv Q, Jiang Y, Qi J, Zhang Y, Zhang X, Fang L, et al. Using mobile apps for health management: a new health care mode in China. JMIR mHealth uHealth. (2019) 7:e10299. doi: 10.2196/10299

57. Mathis F, Vaniea K, Khamis M. Prototyping usable privacy and security systems: insights from experts. Int $J$ Hum Comput Stud. (2021) 38:1-23. doi: 10.1080/10447318.2021. 1949134

58. Yu H, Sun C, Sun B, Chen X, Tan Z. Systematic review and metaanalysis of the relationship between actual exercise intensity and rating of perceived exertion in the overweight and obese population. Int J Environ Res Public Health. (2021) 18:12912. doi: 10.3390/ijerph1824 12912

59. Rabiepoor S, Rezavand S, Yas A, Ghanizadeh N. Influential factors in physical activity amongst pregnant women. Balt $J$ Health Phys Activ. (2019) 11:36-45. doi: 10.29359/BJHPA.11. 2.04

Conflict of Interest: The authors declare that the research was conducted in the absence of any commercial or financial relationships that could be construed as a potential conflict of interest.

Publisher's Note: All claims expressed in this article are solely those of the authors and do not necessarily represent those of their affiliated organizations, or those of the publisher, the editors and the reviewers. Any product that may be evaluated in this article, or claim that may be made by its manufacturer, is not guaranteed or endorsed by the publisher.

Copyright (C) 2022 Yu, He, Wang, Yang, Sun and Szumilewicz. This is an open-access article distributed under the terms of the Creative Commons Attribution License (CC $B Y)$. The use, distribution or reproduction in other forums is permitted, provided the original author(s) and the copyright owner(s) are credited and that the original publication in this journal is cited, in accordance with accepted academic practice. No use, distribution or reproduction is permitted which does not comply with these terms. 